\title{
CESE Report Reversible Cerebral Vasoconstriction Syndrome in Early Pregnancy Treated with Endovascular Therapy
}

Satoru Fujiwara, ${ }^{1}$ Nobuyuki Ohara, ${ }^{1}$ Tomoyuki Kono, ${ }^{1}$ Ryoma Inui, ${ }^{1}$ Hirotoshi Imamura, ${ }^{2}$ Michi Kawamoto, ${ }^{1}$ Nobuyuki Sakai, ${ }^{2}$ and Nobuo Kohara ${ }^{1}$

Objective: We report a case of reversible cerebral vasoconstriction syndrome (RCVS) that occurred during early pregnancy and improved by endovascular therapy (EVT).

Case Presentation: A 30-year-old Japanese woman at 8 weeks' gestation presented with sudden occipital headache followed by left hemiparesis and convulsion. MRI on admission revealed acute infarction in the distribution of the right posterior cerebral artery (PCA), and MRA demonstrated multi-segmental vasoconstrictions in the posterior circulation. Since the episode and image findings were suggestive of RCVS and the patient was in the organogenesis period, intravenous magnesium sulfate was administered as a vasodilator. Her level of consciousness improved temporarily; however, she suddenly fell into a stupor on day 4 of the illness. Emergency MRI demonstrated a fresh infarction in the left side of pons, and the poorer visualization of the posterior circulation. We proposed selective intra-arterial infusion of nicardipine $1 \mathrm{mg}$ over 1 minute through an indwelling microcatheter in the middle of the basilar artery. The left superior cerebellar artery (SCA) and P1 blood flow improved after the procedure. Her symptoms improved gradually, and follow-up MRA performed on day 15 was almost normal. Hence, we established a definite diagnosis of RCVS. She was discharged to recovery phase rehabilitation hospital with modified Rankin Scale 4 and National Institute of Health Stroke Scale (NIHSS) 5.

Conclusion: RCVS can occur in early pregnancy period, and EVT is a potential therapeutic option for RCVS in this condition.

Keywords reversible vasoconstriction syndrome, early pregnancy, endovascular therapy, intra-arterial infusion of nicardipine

\section{Introduction}

Reversible cerebral vasoconstriction syndrome (RCVS) is well recognized as a clinical-radiological entity characterized by thunderclap headache, with or without other acute neurological symptoms, and diffuse segmental constriction

${ }^{1}$ Departments of Neurology, Kobe City Medical Center General Hospital, Kobe, Hyogo, Japan

${ }^{2}$ Departments of Neurosurgery, Kobe City Medical Center General Hospital, Kobe, Hyogo, Japan

Received: December 16, 2019; Accepted: February 17, 2020 Corresponding author: Nobuyuki Ohara. Department of Neurology, Kobe City Medical Center General Hospital, 2-1-1 Minatojimaminamimachi, Chuo-ku, Kobe, Hyogo 650-0047, Japan Email: nobuyuki_ohara@kcho.jp

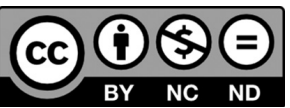

This work is licensed under a Creative Commons Attribution-NonCommercialNoDerivatives International License.

(C)2020 The Japanese Society for Neuroendovascular Therapy of cerebral arteries that resolve spontaneously within 3 months. ${ }^{1)}$ Various related factors with RCVS are reported such as post-partum period, vasoactive drugs, catecholaminesecreting tumors, and other less common clinical conditions. ${ }^{2)}$ However, detailed pathophysiological basis of this condition remains unknown, and therefore, the effective treatment for RCVS has still little evidence. Here, we report a case of RCVS that occurred during an unusual period of early pregnancy treated with endovascular therapy (EVT) (written informed consent was obtained from this patient about this report).

\section{Case Report}

A 30-year-old Japanese woman (gravida 2, para 2) in 8 weeks' third gestation was transferred to our emergency department with sudden onset of left hemiparesis and convulsion after vomiting and headache. She experienced no perinatal complications in the past two pregnancies; 

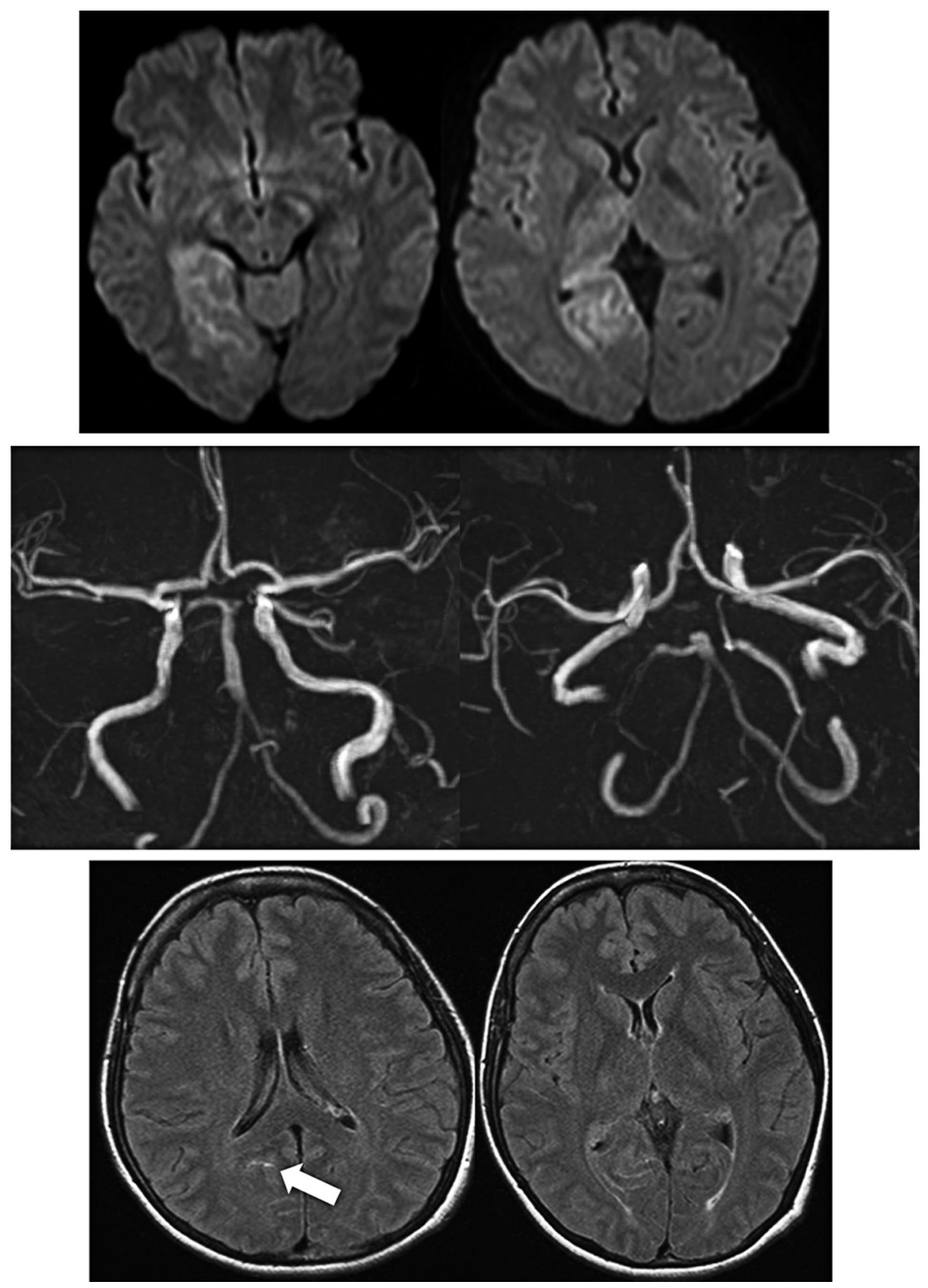

Fig. 1 Magnetic resonance imaging on admission. Diffusion-weighted imaging revealed acute infarction in the distribution of right PCA. MRA demonstrated multi-segmental vasoconstrictions mainly in the posterior circulation, with distal segment of right PCA and left P1 segment was poorly depicted in particular. FLAIR showed thin cortical subarachnoid hemorrhage around right occipital lobe (arrow). FLAIR: fluid-attenuated inversion recovery; PCA: posterior cerebral artery

however, during the first trimester of both, she had morning sickness and experienced sudden occipital headache continued 1 or 2 minutes every time vomiting. On admission, her blood pressure was normal. Neurological examination showed drowsiness, homonymous hemianopia, left hemiparesis, and unilateral spatial neglect, then National Institute of Health Stroke Scale (NIHSS) was 13. MRI revealed acute infarction in the distribution of right posterior cerebral artery (PCA) including thalamus, and thin cortical subarachnoid hemorrhage around the right occipital lobe. MRA demonstrated multi-segmental vasoconstrictions mainly in the posterior cerebral circulation, with left $\mathrm{P} 1$ and right P2-3 segment were poorly depicted in particular (Fig. 1). Since the cerebral infarction also seen in the 

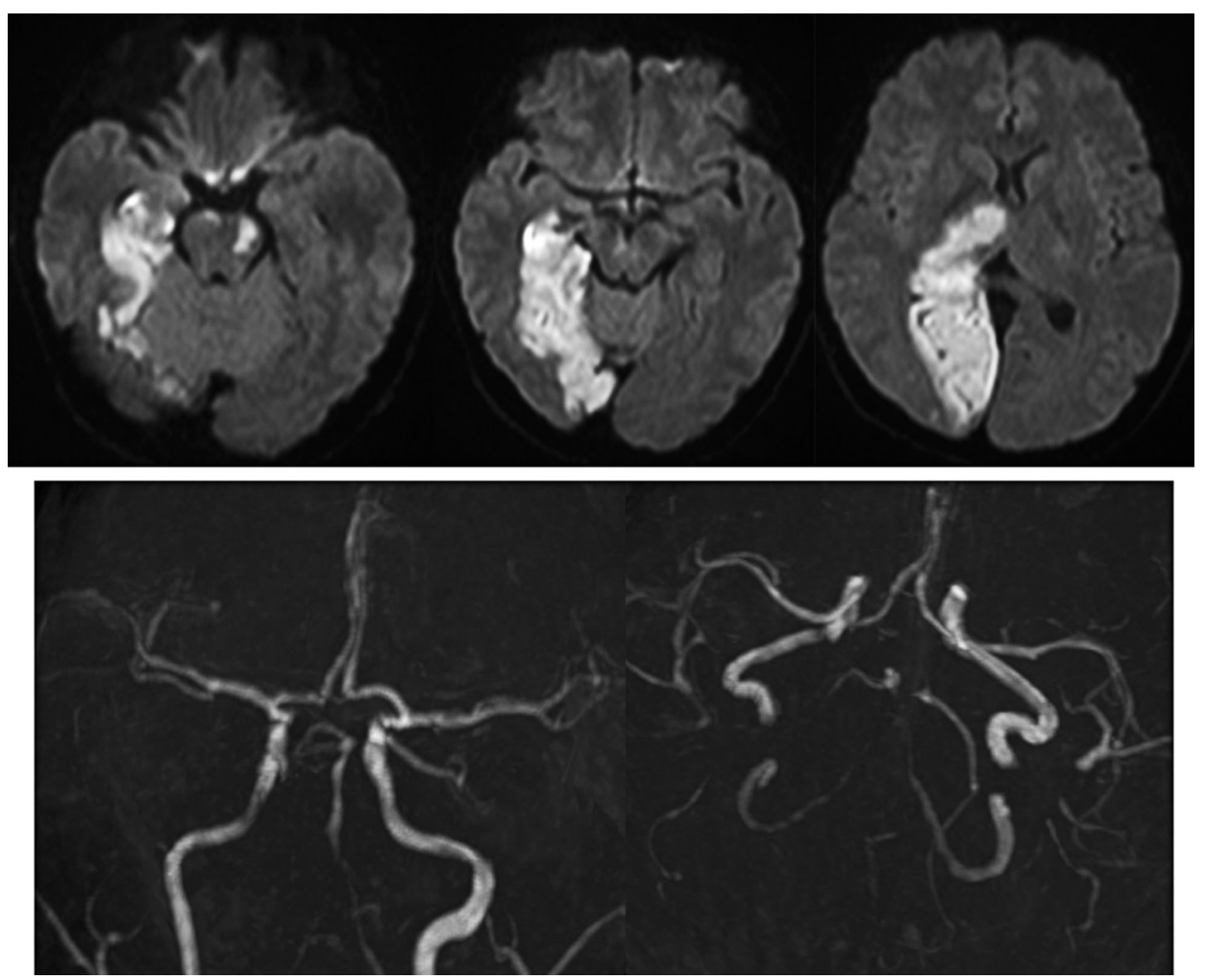

Fig. 2 Imaging findings on the illness day 4. Emergency MRI taken when neurological symptoms worsen demonstrated a fresh infarction in the left side of pons, and the poorer visualization of the diffuse posterior circulation.

right thalamus, it was considered that strong contraction occurred not only in the right P2-3 but also in the P1 at the onset. Cerebrospinal fluid examination showed no evidence of inflammatory diseases and laboratory data were normal including C-reactive protein, erythrocyte sedimentation rate, D-dimer, fibrinogen, activated partial thromboplastin time, prothrombin time, fibrinogen degradation products, and serum catecholamine or estrogen levels. Autoantibodies such as anti-neutrophil cytoplasmic antibodies and anti-cardiolipin antibodies or anti-nuclear antibody were all negative. Since the characteristic episode and image findings were highly suggestive of RCVS, we administered continuous intravenous infusion of magnesium sulfate as vasodilator instead of calcium channel blocker $(\mathrm{CCB})$ and levetiracetam for acute symptomatic seizure. Her disturbance of consciousness once recovered almost completely on day 3 of the illness; however, she acutely fell into stupor again along with pyramidal sign on the right extremities on the following morning. Emergency MRI demonstrated a fresh infarction in the left side of pons, and the poorer visualization of the posterior circulation (Fig. 2). Then, EVT was undertaken for RCVS with medical treatment resistance.
Digital subtraction angiography (DSA) confirmed severe stenosis of the left P1 segment of the PCA and superior cerebellar artery (SCA), whereas the posterior communicating artery supplied to the distribution of the left PCA. Selective intra-arterial infusion of nicardipine $1 \mathrm{mg}$ over 1 minute through a microcatheter (Excelsior SL10 $0.038 \mathrm{inch} / 150 \mathrm{~cm}$, Stryker, Tokyo, Japan) in the middle of the basilar artery improved the blood flow of left SCA and PCA after two cycles of the procedure (Fig. 3). We administered total 5000 units of heparin and $180 \mathrm{~mL}$ of iopamidol. Her abdomen was covered with protectors during procedures, and her estimated radiation absorbed dose was a total of $840.6 \mathrm{mGy}$. Continuous intravenous infusion of nicardipine, regular injection of fasudil, and oral cilostazol and verapamil were administered thereafter, and abortion was done considering the difficulty to overcome her perinatal period with neurological sequelae and the possibility of the relation between pregnancy and RCVS after a careful informed consent with her husband. The operation was completed successfully, then from Day 5 on, her neurological symptoms recovered gradually without recurrence. MRA showed almost normal including posterior circulation on Day 15 (Fig. 4). We established a definite diagnosis of 

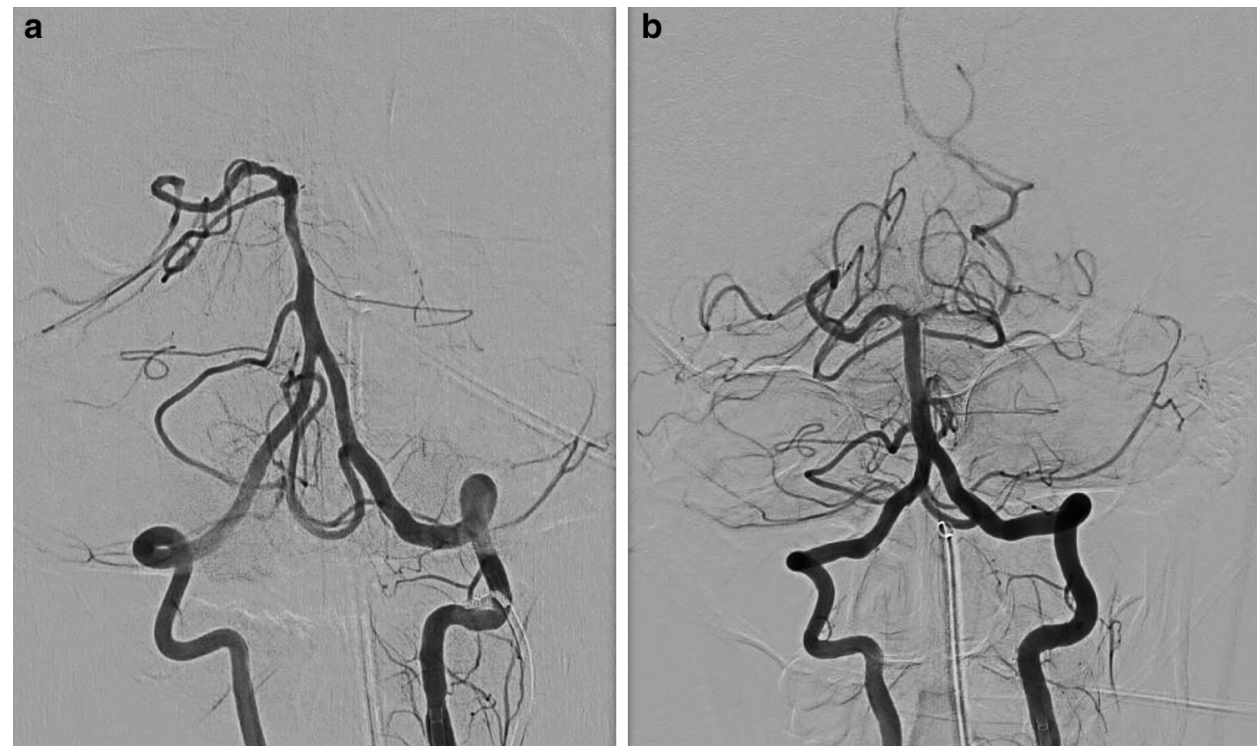

Fig. 3 (a) DSA findings before and after the procedure. Initial DSA confirmed the severe stenosis of left P1 and superior cerebellar artery. (b) After selective intra-arterial infusion of nicardipine $1 \mathrm{mg}$ through an indwelling microcatheter in basilar artery, the blood flow of left P1 and SCA improved. DSA: digital subtraction angiography; SCA: superior cerebellar artery
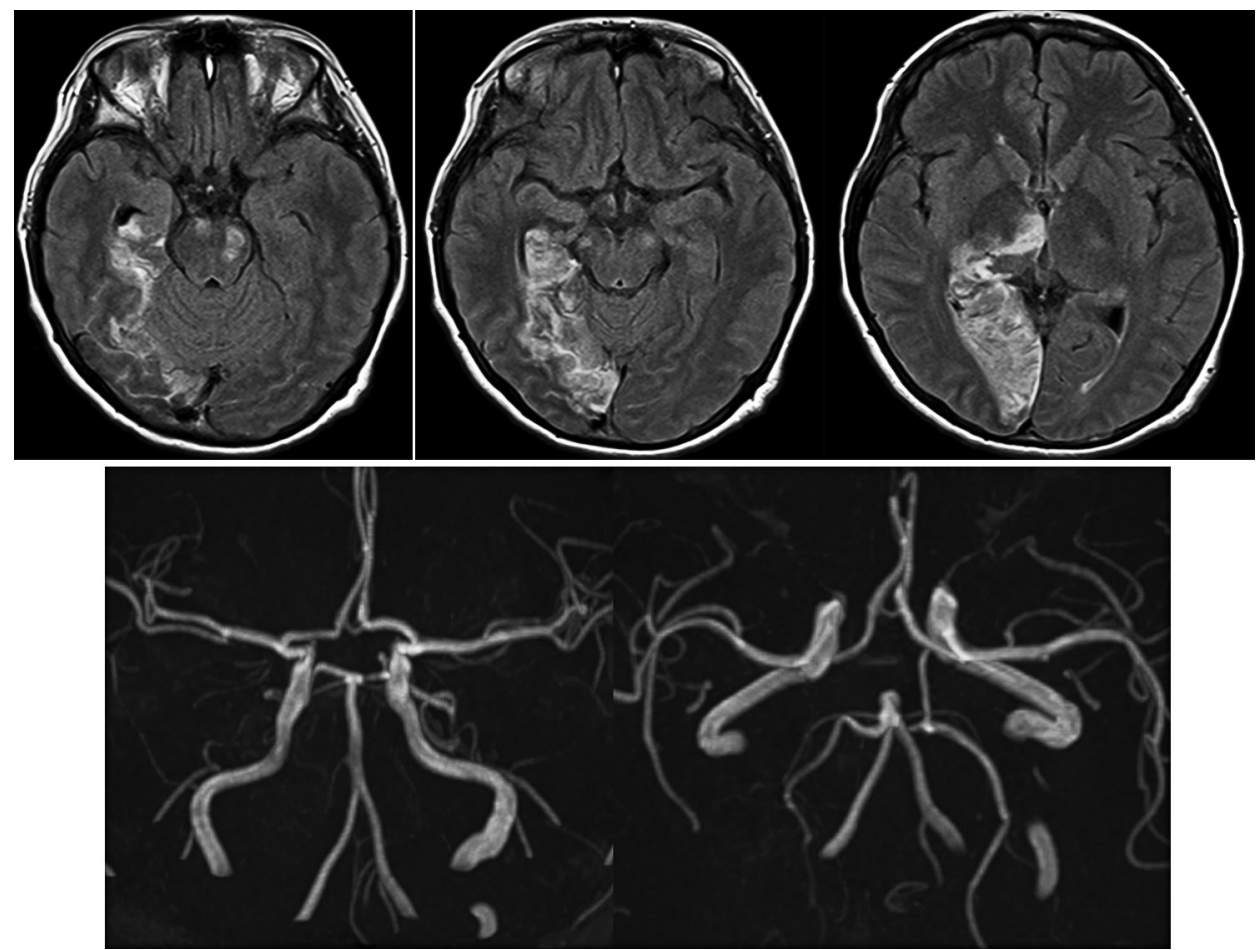

Fig. 4 Imaging findings on the illness day 15. MRA was almost normal including posterior circulation.

RCVS since there was no proof of differential diagnosis of RCVS, such as primary angiitis of the central nervous system, catecholamine-secreting tumors, and hypercarcemia. We speculated that vomiting caused by morning sickness might have triggered thunderclap headache of RCVS. She was discharged to recovery phase rehabilitation hospital on Day 64, with modified Rankin Scale 4 and NIHSS 5 (Fig. 5). 


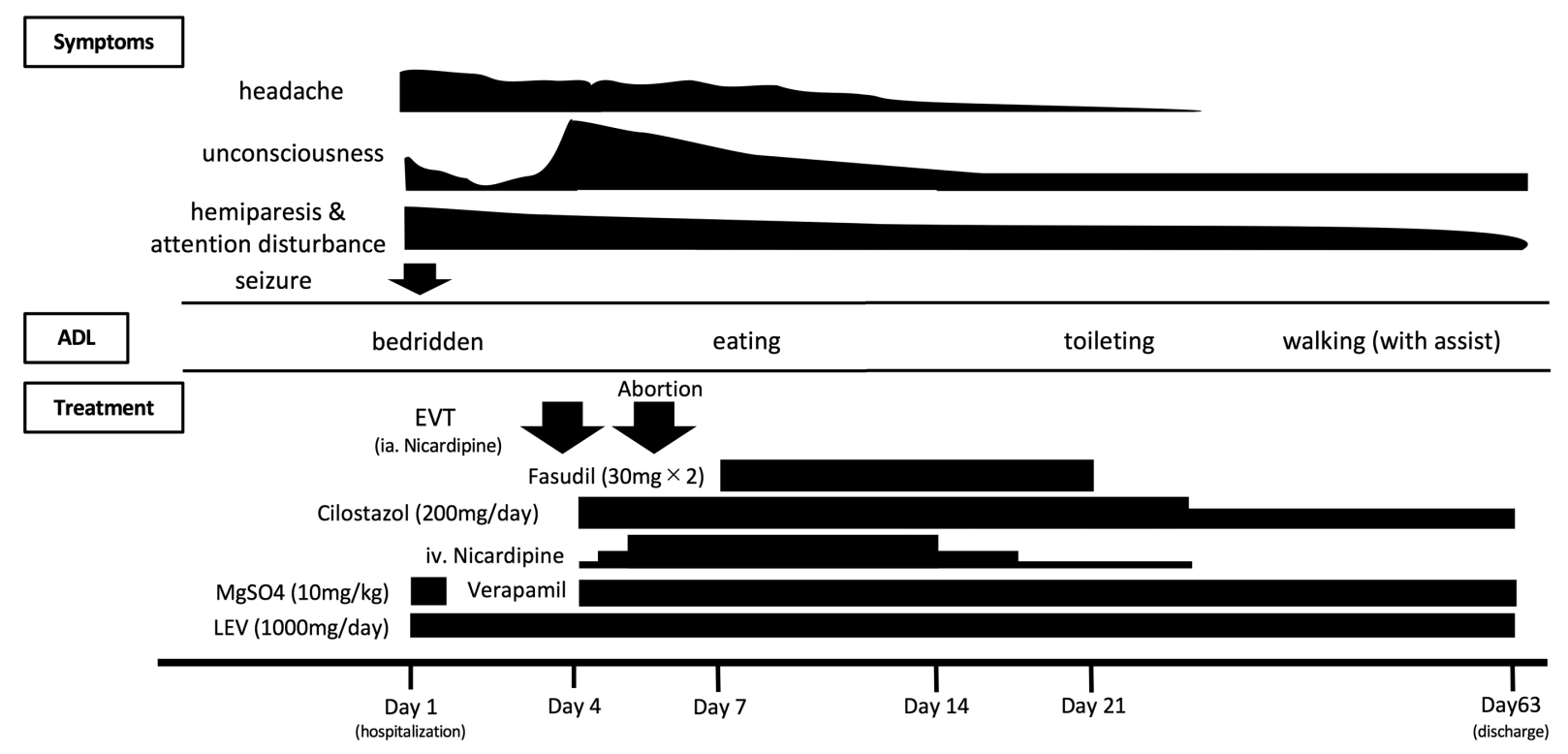

Fig. 5 Clinical course. ADL: activities of daily life; EVT: endovascular therapy; LEV: levetiracetam

\section{Discussion}

In this case, we found two important clinical issues, the first is that RCVS can occur in early pregnancy period, and the other is the efficacy of EVT for the organogenesis period patients. It is well known that a variety of clinical conditions caused RCVS, and a recent review described that sympathetic overactivity, endothelial dysfunction or oxidative stress can lead to vasoconstriction. ${ }^{2}$ Since Valsalva maneuver is a potential cause for sympathetic overactivity, we speculate that repeated Valsalva maneuvers induced by prolonged vomiting might have resulted in cerebral vasoconstriction finally. However, to our knowledge, the similar clinical course of RCVS is not reported in the literature ever.

Second, EVT produced the good response for vasoconstriction. Although several reports have recently addressed the efficacy of EVT such as intra-arterial administration of vasodilators or percutaneous transluminal angioplasty for the treatment of RCVS, ${ }^{3)}$ these procedures for the early pregnant woman were not well documented. Although radiation exposure to the fetus is concern, International Commission of Radiological Protection (ICRP) announced that the fetal radiation risks such as prenatal death, malformation have threshold of $100 \mathrm{mGy}$ or high, so abortion should be considered only when the prenatal doses became above $100 \mathrm{mGy}{ }^{4}$ ) According to a recent study using the standard body phantom, when the head was exposed to a maximum radiation dose of approximately $800 \mathrm{mGy}$, the gonad was exposed to a mean radiation dose of about 0.5 mGy. ${ }^{5)}$ Taken together, therapeutic EVT in brain present no increased risk for fetus, of course we should try to cut down the useless exposure to minimize the risk for cancer that have stochastic effect. Besides, most of the pharmaceutical agents including heparin or iodinate contrast agent and therapeutic devices used in EVT can be utilized in pregnant patients. $\left.{ }^{6}\right)$ As for vasodilator, CCBs such as nimodipine ${ }^{3)}$ and verapamil, ${ }^{7)}$ or milrinone (phosphodiesterase inhibitor) ${ }^{8)}$ have been reported as effective vasodilators for RCVS. In this case, we administered nicardipine, which is a CCB approved and used as an intravenous formulation in Japan, after comprehensively evaluating safety for this patient. As such, it is our belief that EVT should be known as a therapeutic option for progressive RCVS, and pregnancy is not sufficient reason for hesitating the operation in terms of radiation exposure for fetus.

This report has an important limitation. During acute exacerbation phase of RCVS, medical treatments such as abortion and administration of many drugs were performed in addition to EVT; therefore, it is difficult to prove the therapeutic effect of endovascular treatment alone.

\section{Conclusion}

RCVS can occur in early pregnancy period, and EVT is a potential therapeutic option.

\section{Disclosure Statement}

Nobuyuki Sakai received lecture fees from Stryker Japan K.K. and Biomedical Solutions, Inc.; Nobuyuki Sakai 
received a research grant from TERUMO Corporation; Nobuyuki Sakai received consigned research fund from DAIICHI SANKYO COMPANY, LIMITED, TERUMO Corporation, and JIMRO Co., Ltd. The remaining authors have no conflict of interest related to this work.

\section{References}

1) Ducros A: Reversible cerebral vasoconstriction syndrome. Lancet Neurol 2012; 11: 906-917.

2) Ducros A, Wolff V: The typical thunderclap headache of reversible cerebral vasoconstriction syndrome and its various triggers. Headache 2016; 56: 657-673.

3) Ioannidis I, Nasis N, Agianniotaki A, et al: Reversible cerebral vasoconstriction syndrome: treatment with multiple sessions of intra-arterial nimodipine and angioplasty. Interv Neuroradiol 2012; 18: 297-302.
4) The 2007 Recommendations of the International Commission on Radiological Protection. ICRP publication 103. Ann ICRP 2007; 37: 1-332.

5) Tanaka T, Sadatoh A, Hayakawa M, et al: Endovascular treatment of stroke during pregnancy: Measuring the radiation exposure dose of lower abdomen using the human body phantom. JNET J Neuroendovasc Ther 2013; 7: 243-251.

6) Ishii A, Miyamoto S: Endovascular treatment in pregnancy. Neurol Med Chir (Tokyo) 2013; 53: 541-548.

7) Farid H, Tatum JK, Wong C, et al: Reversible cerebral vasoconstriction syndrome: treatment with combined intra-arterial verapamil infusion and intracranial angioplasty. $A J N R \mathrm{Am}$ J Neuroradiol 2011; 32: E184-E187.

8) Bouchard M, Verreault S, Gariépy JL, et al: Intra-arterial milrinone for reversible cerebral vasoconstriction syndrome. Headache 2009; 49: 142-145. 